\title{
Tangence
}

\section{Le décentrement de la modernité littéraire} L'exemple de la Belgique francophone entre les deux guerres

\section{Michel Biron}

Numéro 40, mai 1993

Régionalismes littéraires de la francophonie

URI : https://id.erudit.org/iderudit/025768ar

DOI : https://doi.org/10.7202/025768ar

Aller au sommaire du numéro

Éditeur(s)

Tangence

ISSN

0226-9554 (imprimé)

1710-0305 (numérique)

Découvrir la revue

Citer cet article

Biron, M. (1993). Le décentrement de la modernité littéraire : l'exemple de la Belgique francophone entre les deux guerres. Tangence, (40), 81-99.

https://doi.org/10.7202/025768ar d'utilisation que vous pouvez consulter en ligne.

https://apropos.erudit.org/fr/usagers/politique-dutilisation/ 


\title{
Le décentrement de la modernité littéraire. L'exemple de la Belgique francophone entre les deux guerres ${ }^{1}$
}

\author{
Michel Biron *
}

L'attrait de Paris est une constante de la modernité littéraire belge. S'il se traduit de manière très différente d'une époque à l'autre, il ne faut pas en conclure qu'il varie en lui-même, mais plutôt qu'il s'intègre à des stratégies littéraires qui dépendent étroitement des conjonctures sociale et institutionnelle ainsi que des intérêts personnels des écrivains considérés. Ainsi, les surréalistes belges ne sont pas intrinsèquement plus (ou moins) attirés par Paris que ne l'étaient les symbolistes ou certains naturalistes. Mais la stratégie d'effacement ou de détachement d'un Paul Nougé suppose une représentation de la marginalité qui diffère en substance de celle que pouvaient avoir un Lemonnier ou un Verhaeren. Pour ces derniers, l'accès direct au foyer de légitimation parisien s'avérait possible et déterminait en partie les choix d'écriture. Leur situation périphérique tendait à être surmontée en vue d'une conquête du centre: la "montéee de l'écrivain belge vers Paris était porteuse d'euphorie et d'espoir, la marge quil le localisait était virtuellement et idéalement rayée. Or, ce n'est plus l'oblitération de cette marge que vise Nougé, mais au contraire son exhibition: l'écrivain surréaliste confère à la marge une valeur positive, jouant ainsi sur une triple dissension: par rapport à la société bourgeoise, par rapport à l'institution littéraire en général et par rapport à l'orthodoxie surréaliste en particulier ${ }^{2}$. Se faire

* Michel Biron est professeur à l'Université d'Ottawa. Il a publié plusieurs articles et collaboré au projet Montréal imaginaire.

1 Le contenu de cet article est extrait de ma thèse de doctorat intitulée $L a$ modernité belge. Eléments pour une sociologie bistorique de la littérature en Belgique francopbone, 2 volumes, Université de Liège, Faculté de philosophie et lettres, 1991, 536 p.

2 Cette triple volonté de sécession (sociale, institutionnelle et esthétique) se confirme aussi dans les cas du premier groupe surréaliste * provincial., celui du Hainaut (Achille Chavée, Fernand Dumont, etc.), si l'on admet que l'orthodoxie surréaliste est représentée à leurs yeux par le groupe de Bruxelles. En ce cas, la fidélité à Breton est une manière de refuser la tutelle symbolique de Bruxelles. 
reconnaître par les instances de légitimation parisienne n'a donc pas le même sens selon qu'il s'agit de l'écrivain de 1885 ou de 1925: dans un cas, la quête de la reconnaissance passe par un désir d'équivalence, dans l'autre, par un désir d'altérité.

Pour caractériser les stratégies d'écrivains entre 1920 et 1937 en Belgique, deux ruptures s'imposent tout particulièrement, puisqu'elles résument à elles seules les transformations les plus obvies de la situation de l'écrivain belge. La première, d'ordre idéologique, marque l'obsolescence du mythe unitaire, concrétisée en 1930 par la flamandisation de l'Université de Gand, événement décisif dans l'évolution de la carrière de nombre d'écrivains, en particulier flamands. La seconde, d'ordre linguistique, est la conséquence directe de la première: même s'ils continuent d'écrire en français, nombre de ces écrivains éprouvent quelque honte à œuvrer dans une langue "d'emprunt ", considérée parfois comme étrangère. Cette situation délicate et transitoire n'est cependant pas ressentie également par tous et suscitera des réactions opposées. L'analyse des problèmes d'écriture posés à ce moment-là aux écrivains flamands d'expression française déborde le cadre de ce travail. On aura seulement une idée de ces différences en citant l'exemple de deux Flamands, Franz Hellens et Marie Gevers, qui incarnent des positions antagonistes. Voici un extrait éloquent d'une lettre du premier à l'écrivain surréaliste anversois Paul Neuhuys, datée du 14 mai 1937:

Après votre départ, j'ai réfléchi à ce que vous m'avez dit à propos de Marie Gevers: elle regretterait de ne pas être à même d'écrire en flamand. Ce cas me paraît proprement monstrueux. Un écrivain doit aimer passionnément la langue qu'il écrit.

Marie Gevers aime la littérature, elle n'aime pas la langue qu'elle écrit, parce que cette langue lui paraît en quelque sorte étrangère. C'est le cas de beaucoup d'écrivains belges et ce cas est bien dramatique. Ils ne connaissent aucune langue parfaitement. Comment pourraient-ils aimer et par conséquent manier comme il convient la langue qu'ils écrivent s'ils ne la possèdent pas? ${ }^{3}$

Cette lettre suit de deux mois le fameux *Manifeste du Lundi " où s'est exprimée de la manière la plus retentissante la volonté d'annexion culturelle de la littérature belge d'expression française

3 Lettre de Franz Hellens à Paul Neuhuys du 14 mai 1937, Musée de la littérature (Bruxelles). 
à la France. Or, curieusement, ce texte-phare aux yeux de l'histoire littéraire belge compte parmi ses signataires les noms de Hellens et Gevers, comme si les différences profondes de leurs sentiments relatifs à leur culture d'appartenance s'estompaient devant les nécessités stratégiques de l'heure, comme si "Paris" faisait toujours l'unanimité. Sous l'angle du "Manifeste", analysé plus loin, la diversité des attitudes adoptées par des écrivains tant francophones que flamands se ramène à ce pôle commun, à ce lieu commun qu'est l'attrait de Paris.

Mais le problème ainsi abordé n'est peut-être pas le bon du point de vue de l'histoire sociale et institutionnelle. Entendue dans le sens d'une négation de toute spécificité littéraire belge ou d'un refus du cadre national (régional) au profit de la "véritable " patrie culturelle qu'est la France, la "force centrifuge" qui soustend le "Manifeste du Lundi " est déjà agissante un demi-siècle plus tôt. L'échec de la littérature belge, au sens identitaire et unitaire qu'un discours nationaliste ou patriote lui a accordé, est inscrit au moment même de sa "renaissance", dès l'aggiornamento naturaliste et parnassien des années 1880. Précisément parce qu'elle est mort-née, il semble que cette littérature manifeste une singulière propension à renaître, à s'inventer une autre jeunesse.

Il est vrai que l'époque de la fin du XIX ${ }^{e}$ siècle jouit dans les manuels d'une meilleure existence que celle de l'entre-deuxguerres, mais cela tient plus à Paris qu'à la Belgique elle-même. Si l'historiographie reconnaît le statut de littérature nationale aux textes écrits entre 1875 et 1918, on peut en effet penser qu'elle se fait ainsi l'écho des instances légitimantes parisiennes par lesquelles la Belgique littéraire, par l'intermédiaire surtout du symbolisme, a acquis durant cette période ses titres de noblesse. Certes, il y a un contexte nationaliste en 1890 qui disparait entre les deux guerres, et il faut s'y référer pour comprendre le discours d'accompagnement des œuvres litteraires. Mais la lecture des textes eux-mêmes montre que les mythes désignant en creux la Belgique (l'industrie, le nord, Bruges) se donnent à lire comme des stigmates du national. De Bruges-la-Morte aux Villes tentaculaires, la représentation de la Belgique n'est exploitée que pour être transcendée au nom de valeurs plus hautes, exterritorialisées (la beauté, l'art, la pureté...).

Après une période qui tendait à polariser au maximum les expériences esthétiques, à regrouper les diverses tentatives autour de modèles fortement établis (le Parnasse, le naturalisme, le 
84

symbolisme), les écrivains de l'entre-deux-guerres recherchent toujours la caution symbolique de Paris, mais cette fois par l'éclatement des catégories génériques traditionnelles. Il ne s'agit plus d'écrire comme à Paris, de rivaliser directement avec les écrivains institués, mais d'investir des voies moins encombrées le long desquelles les lois de la concurrence jouent à un bien moindre degré. Au mimétisme affairé d'un Lemonnier succède ainsi la quête de l'étrange d'un Hellens. L'attrait du centre institutionnel se traduit dans un cas par la confrontation esthétique du même (on oppose un naturalisme belge au naturalisme français, un symbolisme belge à un symbolisme parisien) et dans l'autre, par le détour de l'hétérogène. À l'instar du surréalisme, qui émerge en Belgique au moment où il est encore tout à fait marginal à Paris ${ }^{4}$, le renouveau esthétique de la périphérie s'avère alors relativement peu marqué par Paris. C'est ainsi qu'apparaissent dans le royaume des sous-genres tels que le roman fantastique ou le roman policier auxquels on pourrait éventuellement rattacher l'avènement, dans les années 1930, de la bande dessinée.

\section{Roman fantastique, roman policier et bande dessinée}

Plusieurs raisons expliquent l'essor remarquable et durable de la littérature fantastique (ou onirique) en Belgique. Pointer une cause institutionnelle ne doit pas empêcher de voir que le fantastique s'inscrit dans une série littéraire diachronique où s'enchaînent un certain courant romanesque régionaliste, une bonne part des contes et récits symbolistes ainsi que quelques légendes (notamment celle de Thyl Ulenspiegel mise en roman par Charles De Coster). Dans l'introduction de son anthologie du fantastique en Belgique, Jean-Baptiste Baronian écrit que celui-ci, alimenté à la fois au symbolisme et au réalisme, émerge véritablement autour des années vingt, avec Franz Hellens et Jean Ray 5 . Depuis lors, le fantastique est devenu un genre de prédilection des auteurs belges, flamands et wallons, comme en témoignent les œuvres de Michel de Ghelderode, Marcel Thiry, Robert Poulet, Thomas Owen ou, plus récemment, Jean Muno. À cette première explication qui définit une tradition fantastique propre à

4 Ce qui n'était le cas ni du naturalisme ni du symbolisme.

5 Jean-Baptiste Baronian, La Belgique fantastique avant et après Jean Ray, Verviers, André Gérard et Marabout, 1975. 
la Belgique s'ajoute selon Baronian une certaine détermination géographique liée aux paysages uniformes et pérennes:

Et c'est ici sans doute que réside une des grandes raisons d'être du fantastique belge. Contrairement à ce que l'on serait tenté de croire, ce n'est pas dans la pseudo-étrangeté de certains paysages de Flandre ou de Wallonie, dans une improbable géographie de pluie et de brume, qu'il faut le chercher mais plutôt dans le fait que ceux-ci, justement, sont comme figés, éternellement identiques à eux-mêmes, voire rassurants, presque trop sages, trop dociles. ${ }^{6}$

Sous cette causalité diffuse et *atmosphérique", il est permis de voir des causes d'un autre ordre, plus matériel et plus contraignant: le roman fantastique, jouissant d'un crédit symbolique plus faible que le "grand" roman (dominé à l'époque par Gide, Paulhan, Roger Martin du Gard, Rolland, etc.), offre en effet la possibilité d'innover et de se faire un nom à moindres frais.

Par ailleurs, le contournement institutionnel s'effectue au moyen d'autres choix génériques discrédités par la "haute" littérature. C'est le cas du récit policier, genre apparu en France vers le milieu du $\mathrm{xIX}^{\mathrm{e}}$ siècle mais qui reste, à toutes fins institutionnelles, "para-littéraire". L'écrivain le plus international de toute l'histoire de la littérature belge, le Liégeois Georges Simenon, s'en est fait une spécialité. Or, le fait qu'il a commencé sa carrière d'écrivain policier dès après la Première Guerre mondiale, c'est-àdire au même moment où Hellens s'essayait au sous-genre marginalisé du roman fantastique, ne peut pas être considéré comme une simple coïncidence. Les deux expériences relèvent d'une même logique institutionnelle, tendue non plus vers un seul pôle, mais vers plusieurs, jouant sur l'hétérogénéité générique: mélange de réalisme et de fantastique dans un cas, hybridation du psychologique et du policier dans l'autre. Le succès d'abord commercial et, dans une moindre mesure, littéraire de Simenon, sacré meilleur romancier par la figure même de l'institué (André Gide), prouve que le détour par la marge peut éventuellement mener à une certaine forme de légitimité, renforcée depuis lors par l'effet de prestige résultant de l'avènement d'un récit policier sophistiqué (Hammett, Chandler, Robbe-Grillet, etc.). L'émergence de nouveaux noms belges vers la fin des années 1930, tel StanislasAndré Steeman, contribuera à relancer la veine policière.

Ibid., p. 8. 
86

Il paraît peut-être excessif d'associer la naissance d'une bande dessinée en Belgique à la situation institutionnelle décrite précédemment. Le champ de la B.D. et le champ littéraire possèdent des personnels spécifiques et ne visent pas forcément le même public. Pourtant ces deux mondes à part se rencontrent bel et bien en un point de leur institution respective: ils ont chacun affaire soit à l'appareil éditorial en place, soit à des journaux. Ces seules contraintes obligent l'auteur éventuel d'une B.D. à composer avec le dispositif éditorial ou journalistique belge, comme le fait l'écrivain. À la différence du romancier traditionnel cependant, dont l'ambition se porte vers le lieu de consécration obligé qu'est Paris, le bédéiste de l'époque n'a pas de reconnaissance à attendre des instances habituelles, puisque son travail, à la fois scripturaire et pictural, ne s'en remet pas à la seule caution de la littérature. La B.D. tend plutôt à remplacer ou à concurrencer le feuilleton populaire, de toute manière désavoué par la sphère littéraire restreinte. Les avantages matériels évidents qu'en retirent les journaux expliquent qu'une telle pratique esthétique ait pu se développer rapidement en Belgique, où le système éditorial littéraire ne pouvait rivaliser avec celui de Paris. Un nom plus que tout autre incarne cette nouvelle pratique en francophonie: Hergé (Georges Rémi). C'est en effet en 1924 que Rémi publie ses premiers dessins (dans Le boy-scout belge) et entreprend la carrière que l'on sait ${ }^{7}$. Hergé n'a pas inventé un nouveau genre d'expression, mais a repris à son compte une idée déjà populaire aux États-Unis et en France. Avec la création de Spirou en 1938 commence l'âge d'or de la B.D. belge, qui fera école jusque vers 1960 , c'est-à-dire jusqu'à ce que le neuvième art accède à sa véritable consécration par le biais des instances parisiennes ${ }^{8}$. Ainsi, avec la B.D., trois noms émergent à peu près en même temps en Belgique et investissent chacun une forme marginale de la pratique littéraire: Hellens, Simenon et Hergé.

Ces trois auteurs n'ont-ils en commun que le fait d'être nés en Belgique? Il semble en tout cas difficile de les regrouper en

7 :En décembre de la même année, écrivent $D$. De Laet et $Y$. Varende, il y adopte la signature Hergé et y commence à partir de juillet 1926 une courte bande épisodique, "Totor, CP des hannetons", qui marque la naissance officielle de la B.D. belge - (Au-delà du septième art. Histoire de la bande dessinée belge, Bruxelles, Textes et documents, coll. "Chroniques belges:, ministère des Affaires étrangères, du Commerce extérieur et de la coopération au développement, $n^{\circ} 322,1979$, p. 14).

8 Voir Luc Boltanski, : La constitution du champ de la bande dessinée, dans Actes de la recberche en sciences sociales, I, 1975, p. 37-59. 
fonction d'un itinéraire géographique: Hellens et Hergé font carrière en Belgique, Simenon en France. S'il y avait une constellation des écrivains belges de cette époque, elle se reconnaîtrait plutôt à une certaine dissémination des formes littéraires pratiquées. Le surréalisme distant de Nougé s'inscrirait lui aussi dans cette "communauté des bords" de l'institution littéraire. Même "l'inclassable * Michaux, si réticent à l'égard des groupements, suit un itinéraire qui s'apparente, par le choix de formes excentriques, à ces pratiques marginales.

Le rapprochement de ces quelques stratégies d'écrivains concomitantes et inégalement récompensées, menées tantôt ailleurs tantôt sur place, illustre les mécanismes centrifuges à l'œuvre dans l'institution littéraire belge. Il ne s'agit plus simplement de l'attrait exercé par Paris, mais, de manière plus caractéristique, des nouvelles trajectoires choisies par les écrivains de Belgique entre 1920 et 1937 en vue de conquérir un marché. Cette mutation importante des circuits de célébration dans le champ littéraire belge (de grande production comme de production restreinte), lequel se renouvelle désormais par les marges plutôt que par le centre, se produit au moment où se font jour de nouvelles réalités et de nouvelles valeurs. L'idéologie égalitariste associée à la gauche reçoit une certaine légitimité consécutive à la percée socialiste au lendemain de la guerre et contribue à bonifier la valeur du marginal: le suffrage universel adopté en 1919 signifie d'une certaine manière que les exclus du pouvoir constituent le centre de l'électorat de demain. Il s'ensuit que la littérature de masse et les formes d'écriture qualifiées de populaires (légendes, "comicsn, romans policiers, etc.) rachètent leur discrédit esthétique par la nouvelle valeur que leur confère un discours égalitariste auréolé du pouvoir. Par le détour de cette idéologie, le quantitatif n'est plus réductible au mercantile.

À ce point de l'analyse, d'autres facteurs historiques pourraient être rattachés à l'émergence de nouvelles formes d'écriture: l'événement le plus important est sans doute celui des deux guerres mondiales, pendant lesquelles la circulation souterraine, clandestine, de la culture est glorifiée (c'est ce qui échappe au pouvoir illégitime d'occupation qui sera reconnu par l'histoire); le krach de 1929 révèle d'autre part la fragilité du système économique occidental et exhibe par l'image (le film Misère au Borinage de Henri Storck date de ces années) la misère des classes inférieures belges. Révolution politique, guerre et crise économique 
agissent indirectement sur un certain éclatement des pratiques littéraires.

\section{Franz Hellens}

L'exemple le plus connu de ce décentrement esthétique s'appelle Franz Hellens. Écrivain prolifique, proche des avant-gardes mais plus encore des modernistes, Hellens est l'une des figures les plus mobiles du milieu littéraire belge d'alors. L'historien littéraire Marc Quaghebeur, après avoir noté son influence successive sur Michaux et Ghelderode, compare le trajet hellensien à celui de Lemonnier un demi-siècle plus tôt:

La cohérence du dessein comme sa pertinence par rapport aux mentalités des écrivains du temps vont assurer à Hellens, pendant cinquante ans, une place équivalente à celle de Lemonnier durant la période précédente. Véritable carrefour des idées et des talents, polygraphe aussi impénitent que son aïeul trapu des années léopoldiennes, esprit curieux des divers aspects de son temps et capable de les formuler intellectuellement, travailleur inlassable mais novateur modéré, Hellens devient ainsi très vite la véritable éminence grise de nos lettres. ${ }^{9}$

Le rapprochement de Hellens et de Lemonnier est instructif à plus d'un égard. Figure consacrée dans la convivialité des années 1880 en Belgique, le "Maréchal des lettres* (Lemonnier) est aussi celui qui r'a cessé de chercher sa place en littérature, perpétuel déplacé. Son naturalisme teinté de régionalisme porte les traces de sa quête inachevée et de la fragilité de la structure institutionnelle en Belgique. Si la position de Hellens rappelle la sienne, elle participe d'une stratégie de mobilité qui diffère pourtant du mimétisme de Lemonnier. Son cuvre se caractérise surtout par une étonnante diversité des formes, qui conduit parfois à la fusion générique. Après avoir publié un premier roman écrit dans le style d'Eekhoud et de Lemonnier avec un titre qui fait écho à Rodenbach, En ville morte (1906), il se fait remarquer par ses aînés grâce à quelques récits et contes. Au sortir de la guerre, il est âgé de 37 ans et possède un manuscrit qu'il a rédigé durant

9 Marc Quaghebeur, *Balises pour l'histoire de nos lettres *, dans Alphabet des lettres belges de langue française, Bruxelles, Association pour la promotion des lettres belges de langue française, 1982, p. 55. 
ses séjours sur la Côte d'azur entre 1916 et 1917, Mélusine. En l'espace de deux ans, il publie coup sur coup, outre cet étonnant roman, les courts récits oniriques de Nocturnal (1919), le poème La femme au prisme (1920), un autre roman intitulé En écoutant le bruit de mes talons (1920) et crée en 1921 l'importante revue Signaux de France et de Belgique. Parallèlement à cette production frénétique, notons le renversement d'attitude de Hellens au sujet du statut de la littérature de Belgique. Raphaël de Smedt a montré que c'est à partir de la fin de 1919 que l'auteur de Mélusine s'est opposé à l'idée d'une spécificité nationale des lettres belges ${ }^{10}$. De l'article qu'il publie alors pour applaudir à l'entrée de Maeterlinck à la Comédie-Française jusqu'au "Manifeste du Lundi * dont il est un des promoteurs les plus actifs, il semble qu'il y a une étroite filiation.

Avant d'examiner le sens et la portée du "Manifeste", il convient de s'arrêter un moment sur ce tournant dans l'évolution de la carrière de Hellens et en particulier sur ce curieux roman qu'est Mélusine. Publié en 1920 à l'enseigne d'une société bruxelloise (le Voile rouge) après avoir été refusé par différents éditeurs parisiens, Mélusine ne fut jamais lancé à Paris malgré une entente en ce sens avec l'éditeur Émile-Paul. Il s'agit donc d'un roman tout à fait inconnu dans l'Hexagone jusqu'à sa réédition en 1952 (avec de très nombreuses corrections de la part de l'auteur) ${ }^{11}$. Le roman n'en eut pas moins de très élogieuses critiques dans les milieux avant-gardistes belges. Paul Neuhuys n'hésite pas à écrire dans Ca ira de septembre 1921:

C'est Franz Hellens qui a l'heure actuelle semble incarner le plus complètement les qualités de notre race. Il a atteint la pleine possession de ses moyens. Son lyrisme ne s'interdit plus aucune liberté et un roman tel que Mélusine marque incontestablement une brillante apogée dans l'œuvre d'un écrivain. ${ }^{12}$

10 Raphaël de Smedt, « Franz Hellens et le problème des lettres françaises de Belgique, dans Michel Otten (dir.), Études de littérature française de Belgique offertes à Josepb Hanse, Bruxelles, Jacques Antoine, 1978, p. 192.

11 Sans la moindre influence en France, partant sans avoir été consacré par les instances parisiennes, ce roman attend aujourd'hui encore ses exégètes. Se mesure ici le poids de la caution critique autorisée, qui commande non seulement l'activité des écrivains, mais aussi celle des lecteurs, universitaires ou non.

12 Ca ira, septembre 1921, p. 82. 
Henri Michaux fournira sans doute l'hommage le plus remarquable lorsqu'il reconnaîtra que, sans Mélusine, il n'eut pas songé à écrire son premier ouvrage, Essai philosophique et littéraire. Les rêves et la jambe (Ca ira, 1923) ${ }^{13}$.

Qu'on puisse rattacher Mélusine à Michaux, à Freud, au fantastique, au surréalisme, au dadaïsme, au cinéma et à la bande dessinée ${ }^{14}$ atteste son caractère hétérogène. Le roman est divisé en vingt-six chapitres, tous maintenus lors de la réédition de 1952. Â l'exception des deux chapitres liminaires, qui assurent la cohérence de l'ensemble, la plupart des séquences narratives sont des segments autonomes et permutables, intitulés souvent de manière délibérément infantile: «je vais chercher Mélusine au bal de l'Opéra *, "Mélusine s'habille ", "Mélusine fait du Music-Hall ", etc. La référence à la fée Mélusine, telle que représentée depuis le Moyen-Âge, n'éclaire qu'en partie les enjeux du roman. Jean d'Arras raconte dans son roman en prose écrit en 1392 ou 1393 l'histoire des Lusignan, d'où est issue la mère Lusigne qui deviendra Mélusine (après Goethe, de nombreux poètes français du XIX ${ }^{e}$ siècle, par exemple Hugo et Nerval, y ont fait allusion). La légende veut que tous les samedis soirs, la fée Mélusine voyait le

13 Michaux écrit: ‘ $\mathrm{A}$ la première lecture, je devinai que c'étaient des rêves, quoique Mélusine ne soit pas présentée comme tel. L'auteur par la suite confirme mon intuition. . Il ajoute: ‘Que Mélusine comprenne une satire, un éloge de la vie moderne, c'est possible. Mais ce que Mélusine a dans le ventre, c'est cette originalité, c'est l'énergie inouie, du style morceau d'homme, du style rêve. (p. 24-25). La distinction que fait Michaux entre l'éloge de la vie moderne et le style rêve. recoupe une division déjà perçue entre le modernisme (être pour la société nouvelle) et la négativité qui est plutôt le fait de l'avant-garde (le style rêve* pointant une distance par rapport à la réalité sociale). Par des romans composites comme Mélusine et par son activité ultérieure de directeur de revue d'avant-garde, Hellens semble vouloir concilier les deux attitudes.

14 Le rapport à Freud est explicitement suggéré par Michaux; l'écriture fantastique sera l'une des constantes de l'écriture de Hellens; le surréalisme prémonitoire est évoqué par plusieurs, dont Robert Frickx et Robert Burniaux (La Littérature belge, Paris, P.U.F., coll. "Que sais-je?", p. 55); la dimension dadaïste est mentionnée par Paul Gorceix dans la préface de la réédition chez Jacques Antoine (coll. "Passé Présent , 1987, s.p.); le même critique affirme plus loin: * Dans cette aventure onirique dont plus d'un épisode préfigure la B.D.»; enfin, Robert Frickx parle ailleurs de l'influence évidente de l'écriture cinématographique, le nom de "Locharlochi * étant l'anagramme de Charlot (Lettres françaises de Belgique. Dictionnaire des oeuvres I, Le roman, Paris-Gembloux, Duculot, 1988, p. 326). 
bas de son corps muer en peau de serpent; tel ne sera pas le cas du personnage éponyme de Hellens, bien qu'il participe lui aussi du mythe de la chute. D'entrée de jeu, une immense cathédrale se dresse en plein désert d'Afrique devant Mélusine et le héros narrateur; après qu'ils ont vainement essayé d'y entrer par le sommet, la cathédrale glisse sous leurs pieds et disparaît d'un coup, les laissant seuls dans leur "chute. Commence alors une longue quête dans laquelle les adjuvants et les opposants se multiplient et s'échangent leurs rôles tandis que l'objet (au sens actantiel) se transforme: après la cathédrale perdue, c'est une robe de saphir, perdue par Mélusine en "tentant fortune ", qu'il s'agit de retrouver. Le péché du jeu, version moderne du péché originel, laisse le narrateur, qui s'était déshabillé lui aussi pour jouer, "plus honteux de [sa] nudité qu'au départ"15. Afin de retracer la robe de saphir (qui donnera le sous-titre du roman en 1952), le narrateur cherche d'abord secours auprès d'un ingénieur, Nilrem, celui-là même qui les avait guidés après leur "chute " de la cathédrale. Personnage légendaire (son nom est le boustrophédon de Merlin l'enchanteur) autant que fabricant de légendes, Nilrem tient un rôle dont la duplicité apparaîtra bientôt au narrateur, qui ne sait plus à qui faire confiance. Nilrem enchante mais, selon son étymologie latine, il annihile (*rienchose ") aussi, télescopant l'enchantement et le rien, projetant le merveilleux dans l'absurde. Promené par l'ingénieur de jardins fantastiques en villes extraordinaires, le narrateur finira par recourir aux services d'un détective, appelé "CEil-de-Dieu ". Ce personnage picaresque (et de la famille de Caïn), à la fois voyant et dupe, bravant tous les dangers sans les voir et prenant le ridicule au sérieux, introduit une nouvelle dimension générique dans l'œuvre de Hellens: celle du récit policier, mais un récit sans aucune crédibilité ${ }^{16}$. Aux confins de l'onirique, du fantastique et du policier picaresque, Mélusine entremêle aux images poétiques (telle la cathédrale en plein désert) une série d'événements diégétiques sans suite. Surchargé, interminable dans sa surenchère (surtout dans sa première mouture), ce conte romanesque à multiples entrées génériques transporte le plus ancien (la légende de Mélusine, la référence biblique, le style picaresque, etc.) dans

15 Franz Hellens, Mélusine, Bruxelles, Jacques Antoine, coll. "Passé Présent *, 1987, p. 105.

16 . CEil-de-Dieu * donnera son nom à un roman de Hellens paru en 1925 et prolongeant cette veine du policier picaresque. 
92

l'univers composite de formes nouvelles. Occupant les bords de l'institution littéraire, cet ambitieux récit de Hellens donne à voir un texte ouvert aux dérives esthétiques et totalement dépourvu de centre.

\section{Le Manifeste du lundi (1937)}

Le 1er mars 1937, un groupe d'une vingtaine d'écrivains belges, habitués à se réunir le premier lundi de chaque mois dans un restaurant de Bruxelles ou à la Maison d'Art, publie un manifeste de six pages pour réfuter l'idée selon laquelle les textes littéraires produits par des citoyens belges formeraient une littérature belge. Dissociant la patrie culturelle et le pays politique, les signataires (issus surtout de Flandre, mais aussi de Wallonie et de Bruxelles) réclament que *la Belgique de Maeterlinck et de Baillon [fasse] partie intégrante de cette entité, indépendante de toutes frontières, qu'est la France littéraire "17. L'argument invoqué pour preuve de ceci est fondé sur le fait que tous les textes français de Belgique se rattachent aisément à un épisode de l'histoire de la littérature française: Rodenbach, Verhaeren, Mockel et Van Lerberghe se réclament du symbolisme, Girard et Séverin du Parnasse, Van Hasselt et Pirmez du romantisme, etc. Ce jeu d'équivalence culturelles implique la mise à l'écart du fait politique et institutionnel et relève plus que jamais d'une volonté d'émancipation de l'écrivain périphérique:

S'il est vrai, d'autre part, que l'indépendance politique de la Belgique constitue pour nos lettres, à travers diverses circonstances morales ou pratiques, un facteur notable de "localisation*, il tombe sous le sens que les conditions essentielles de la création littéraire ne sont pas différentes dans notre pays de ce qu'elles sont dans n'importe quel pays de langue française.

La faiblesse de l'argumentaire (à peine dêfendable vu la généralisation finale qui néglige les différences profondes des conditions de création en Belgique, à Haïti, au Maghreb ou au Québec) n'a pas empêché le "Manifeste " d'emporter l'adhésion de la plupart de ses commentateurs. Car si la sociologie récuse sans hésiter le postulat de départ des Lundistes (uniformité des conditions d'écriture dans la francophonie), (presque) toute l'historiographie littéraire belge tend à lui donner raison.

17 Le Manifeste du Lundi, Bruxelles, Impr. Van Doorslaer, 1937. 
D'un point de vue diachronique, il est à remarquer qu'au fond le "Manifeste " ne procède pas autrement que Verhaeren ou Rodenbach: il désigne la Belgique à la France en marquant de manière explicite la défaveur de la première par rapport à la seconde. Cette dévalorisation, l'écrivain belge ne "l'attend" pas de Paris: il l'anticipe et l'infuse dans sa propre revendication. Comme le *Nord " industriel et tentaculaire de Verhaeren racheté par Paris et Florence, comme la Bruges inanimée de Rodenbach tournée en mythe de l'art par l'écriture symboliste, les Lundistes nomment la Belgique pour mieux s'en détacher. Dire et médire participent d'un même geste, dont les dividendes symboliques ne sont que trop visibles dans le cas du "Manifeste. David Scheinert, auteur d'Écrivains belges devant la réalité (1964), en témoigne malgré lui lorsqu'il écrit, à propos de cette mouvance:

À quoi tient [...] cette volonté de prendre ses distances par rapport à la réalité première, le milieu familier, peuple et pays? À plusieurs raisons dont la plus importante n'est pas le désir de se faire éditer en France ou ailleurs. ${ }^{18}$

L'itinéraire de Charles Plisnier, signataire du "Manifeste", illustre au mieux cette quête de la reconnaissance parisienne que suggère, tout en l'écartant pudiquement, Scheinert. Après son exclusion du Parti communiste en 1927, Plisnier se consacre activement à la vie intellectuelle et lance même une sorte de salon littéraire en 1929, connu sous le nom des Mardis de la Place Morichar. Pendant neuf ans, il recevra ainsi une foule hétérogène d'artistes, d'écrivains et d'intellectuels, jusqu'à son départ pour Paris au printemps de 1937. Entre-temps, il publie divers romans et recueils, dont quelques nouvelles dans les Feuillets bleus, revue liégeoise faussement domiciliée à Paris. Le caractère anecdotique de sa montée à Paris et de la consécration (très provisoire) que lui vaut l'obtention du prix Goncourt (1937) ne doit pas empêcher de voir dans cet épisode de la vie littéraire belge une mise

18 David Scheinert, Écrivains belges devant la réalité, Bruxelles, La Renaissance du livre, 1964, p. 11. La cause est plus profonde: Après tant de bouleversements politiques, beaucoup d'écrivains ou d'artistes déçus à la fois par l'ancien régime et par la révolution, ont le sentiment de s'être perdus, de n'être plus eux-mêmes. Ils pensent que c'est la société - n'importe quelle société - qui est responsable de cette aliénation. "Lauteur insiste sur le rejet conséquent du politique par l'écrivain belge: a Pour ces écrivains, la politique est la grande ennemie, celle qui altère la personnalité de l'individu.. 
en acte révélatrice des propos du "Manifeste". Résumons d'abord avec Véronique Jago-Antoine le film de ce qu'elle appelle les "Tribulations d'un prix Goncourt " ${ }^{19}$. Conscients d'avoir en mains avec Mariages (1936) un roman "goncourable ", Plisnier et sa maison d'édition (Correa) attendent avec impatience le verdict des Dix, pour qui l'élection d'un écrivain de nationalité non française serait une première. La mise hors concours du Belge en vertu de sa citoyenneté provoque la colère du milieu littéraire bruxellois, qui organise sur le champ un banquet. Projeté pour la première fois sur le devant de la scène littéraire, Plisnier profite du bruit de son éviction pour faire fructifier cette soudaine notoriété en capital symbolique: après s'être fait un nom à Paris par l'aventure ratée de Mariages, il y séjourne deux semaines puis installe sa famille en banlieue, à Saint-Germain-en-Laye:

Outre qu'elle secrète ce goût de province auquel Plisnier sera toujours sensible, l'aire est stratégique: offerte aux regards des instances légitimantes de l'institution française, elle préserve le candidat de l'oubli où s'enlisent nombre d'écrivains provinciaux. 20

Il publie alors Faux passeports, collection de nouvelles qui, à l'exception de la dernière, sont toutes déjà parues ailleurs. Ses efforts seront rapidement récompensés, par l'obtention in extremis du Goncourt d'abord, puis par une succession de gratifications symboliques et matérielles, la plupart obtenues en Belgique $^{21}$.

Des Mardis de la Place Morichar aux dîners du Lundi, de Mariages et Faux passeports au "Manifesten, la figure de Plisnier semble chargée des ambitions littéraires d'une partie des écrivains belges actifs en 1937 et lorgnant vers Paris. Toutefois, il ne suffit pas de constater l'attrait qu'exerce la métropole et que le "Manifeste" avoue en même temps qu'il l'avalise, phénomène inhérent

19 Véronique Jago-Antoine, :Tribulations d'un prix Goncourt", dans Paul Aron (dir.), Charles Plisnier. Entre l'Évangile et la Révolution, Bruxelles, Labor, coll. a Archives du futur", 1988, p. 11-18.

$20 \quad$ Ibid., p. 13.

21 Il est, entre autres, nommé Chevalier de l'ordre de Léopold. Il entre aussi au même moment à l'Académie Royale de Langue et de Littérature françaises de Belgique (la décision avait été prise avant l'annonce du Prix Goncourt, mais l'effet d'anticipation n'est pas exclu étant donné que l'Académie savait l'écrivain en lice). Enfin, il devient directeur de collection chez son éditeur Correa. 
à la modernité littéraire belge. Plus intéressante est la manière dont, en 1937, Plisnier parvient aux fins qu'il s'était proposé d'atteindre. Considérons un moment les deux romans qui ont permis son succès: le premier des deux, Mariages, de facture réaliste traditionnelle (contrairement au récit poétique publié en 1933, L'enfant aux stigmates), est un roman qui conjugue le social et le psychologique. Il a été noté par plusieurs que l'action s'y déroule dans le nord de la France alors même que les lieux décrits évoquent sans jamais la nommer Mons, ville natale de l'auteur. Le récit transpose ainsi une réalité géographique de la province belge du Hainaut dans une onomastique hexagonale. Si l'on suit par exemple José Fontaine, on conclura que la stratégie de Plisnier était de taire sa nationalité pour augmenter ses chances d'être couronné par le jury du plus important prix littéraire de France ${ }^{22}$. En admettant que Mariages réponde ainsi à l'injonction du «Manifeste *, à savoir l'annexion de la Belgique littéraire francophone à la patrie culturelle française, il faudrait cependant considérer Faux passeports comme une incongruité. En effet, après l'échec de la première stratégie (roman s'inscrivant fortement dans la tradition réaliste française et substituant même les noms de lieux pour "faire" davantage français), l'auteur choisit une tactique inverse: présenter un roman sous forme de nouvelles détachées où, malgré un avertissement sans équivoque ("le je de ce livre n'est pas moin), la vie militante de Plisnier dans le P.C.B. est narrée de manière presque autobiographique. Or, chronologiquement, ce n'est pas Mariages mais Faux passeports qui lui vaut le Goncourt, ou, plus exactement, c'est par la mise en relation des deux textes que Plisnier se voit couronné. Véronique Jago-Antoine a raison de souligner que *c'est un écrivain de l'engagement que Paris choisit de consacrer en ces temps troublés d'avant 39 , au moment où la Belgique va se résoudre à la neutralité.23. Les Dix n'ont pas seulement voulu réparer l'indélicatesse de l'année précédente; il serait douteux que le seul regret d'être passé à côté d'une *grande » œuvre ait convaincu une majorité d'entre eux de revenir sur une décision antérieure. En d'autres mots, il ne semble pas que l'instance de consécration parisienne ait eu intérêt à récompenser un roman étranger dont la marque étrangère était absente. Faux passeports, c'est l'envers de

22 José Fontaine, „Plisnier et la question nationale, dans Paul Aron (dir.), op. cit., p. 116-117.

23 Véronique Jago-Antoine, loc. cit, p. 16. 
Mariages, c'est la nouvelle opposée au roman, le «je "opposé au *il n et l'identification au communisme opposée à la dénonciation distante de l'expérience bourgeoise du mariage. L'écrivain périphérique n'est admis dans le cercle des initiés qu'après s'être déclaré " périphérique ", muni d'un " faux passeport " le désignant à la fois semblable et différent pour le lecteur hexagonal. Il s'ensuit que la relation de l'écrivain lundiste vis-à-vis de son pays ne se ramène pas à une simple dénégation: le contexte belge se trouve à la fois désigné et infériorisé par la combinaison de ces deux œuvres.

De son côté, le "Manifeste du Lundi relève d'une duplicité comparable, dont le ressort est encore une fois la visée parisienne. À la suite de Robert Vivier, Marc Quaghebeur remarque pertinemment que "la position du "Manifeste" désigne les destinataires du document comme étant la communauté culturelle parisienne plus que l'opinion belge "24. En se voulant une véritable demande d'annexion à la classe littéraire française, le *Manifeste ne procède pas autrement que les deux romans de Plisnier: après avoir décrit la situation d'énonciation et le problème du nom belge (et du "non-Belge"), il projette une image infériorisée du milieu littéraire local. La construction même du texte répond en tous points à ce qui ressemble de plus en plus à un protocole structurel des rapports de l'écrivain belge à Paris, comportant deux phases indispensables: la dénomination et la dévalorisation. La première phase comprend le paragraphe liminaire où est nommé le "Groupe du Lundi * et la partie qui suit, intitulée «Qu'est-ce que les Lettres belges?». Paradoxalement, le texte continue à se référer à ce qu'il prétend dénoncer, à savoir l'existence des "Lettres Belges". La seconde phase occupe la majeure partie du "Manifeste", la mise en mots des difficultés propres à la condition littéraire belge étant aussi une mise à distance. On y reproche notamment aux campagnes en faveur du livre belge de susciter "la louange systématique, l'esprit de camaraderie vulgaire, l'égalitarisme grossier et son corollaire, l'horreur de toutes les indépendances, de toutes les originalités et de toutes les supériorités, la tendance au grégarisme». Tous les termes employés partagent un idéologème: celui de la communauté refusée, enté sur une chaîne isotopique fondée sur le sème négatif du

24 Marc Quaghebeur, "Balises pour l'histoire de nos lettres *, dans Alphabet des lettres belges de langue française, op. cit., p. 57. 
commun (synonyme de tribalisme et impliquant des faveurs personnelles): "systématique ", "vulgaire ", "grossier ", "égalitarisme", refus des *indépendances", des *originalités " et des "supériorités ", "grégarisme ". Le *nous" contre-signé par les vingt et un écrivains appelle à la désunion: il est un *je " déguisé, une addition de personnes refusant l'idée de groupe et, par conséquent, la situation d'énonciation du "Manifeste" lui-même (par définition, une affaire de groupe). Que les écrivains en présence n'aient rien de commun confirme ce désaveu général du "groupe " au profit de l'un, de l'individu, du distinctif. L'un des mécanismes institutionnels mis en lumière par des sociologues de la littérature en Belgique au début des années $1980^{25}$ trouve peut-être ici sa source: Paris n'admet les agents périphériques que s'ils renoncent au préalable à toute prétention commune. $\mathrm{La}$ stratégie individualiste des Lundistes s'est depuis ce temps imposée à une partie importante des écrivains de Belgique ambitionnant d'accéder à la sphère littéraire restreinte de Paris.

Fait significatif, qui confirme la destination française du -Manifeste *, la dévalorisation est toujours impersonnelle, s'appliquant à l'ensemble du milieu littéraire belge, sans plus de précision. Impossible, par exemple, de savoir qui est visé en réalité par les accusations suivantes:

La vie littéraire se réduit chez nous, d'une part à une pompe académique, respectable en soi, mais sans aucune autorité fondée sur des noms et des œuvres, et d'autre part à un associationnisme puéril, où l'on voit l'amateur coudoyer l'homme de métier et la mauvaise littérature faire pièce à la bonne. Ajoutons à cela un gérontisme tellement obstiné que les écrivains belges de soixante ans et plus, salués ailleurs comme des maîtres, font encore figure dans leur patrie de quasi débutants, quotidiennement raillés et discutés.

De quelle "pompe académique * s'agit-il? Charles Bernard, Georges Marlow et Horace Van Offel font déjà partie de l'Académie royale de langue et de littérature françaises de Belgique, et de nombreux autres signataires s'y destinent. À quoi se réfère

25 . Pour des agents périphériques, la "scène parisienne" n'est accessible qu'à des outsiders qui ne sont reconnus que parce qu'ils n'ont qu'une caractéristique commune: celle de n'avoir rien de commun. (Michel Condé, Pierre Popovic et Marie-Paule Remacle, L'édition du livre dans la communauté française de Belgique, Institut de sociologie de l'Université de Liège, Commission Art et Société, 1980, p. 87). 
98

"l'associationnisme puéril *? On ne le sait pas (la *communauté" semble visée de manière générale). Quant au gérontisme, l'allusion est ambiguë: vu le nombre élevé de sexagénaires chez les Lundistes (Charles Bernard, Grégoire LeRoy, Georges Marlow, Camille Poupeye, Henri Vandeputte), il semble qu'on regrette surtout l'absence de reconnaissance digne de leur rang. Reste la question du régionalisme, dont tous prétendent se distinguer résolument: il s'agit du repoussoir le plus explicite du "Manifeste *, dont l'ambition est précisément de déborder la frontière du marché littéraire régioral, mais aucun auteur régionaliste (au sens péjoratif) n'est cité nommément.

$\mathrm{Au}$ total, la valeur polémique du *Manifeste * est relativement ténue: personne n'est pris à charge directement, les groupes dénoncés n'ont pas de répondant dans la réalité (sauf à considérer qu'il s'agit justement des groupements auxquels on est tenté de rattacher plusieurs signataires), la seule esthétique décriée (le régionalisme) n'est illustrée d'aucun exemple et, en définitive, aucun engagement n'est requis de la part des signataires. Le "Manifeste " se termine en formulant le vœu que "notre" littérature revête l'aspect qui lui convient et tienne *la place qu'elle mérite au sein des lettres françaises ". L'ambiguité de cette conclusion, qui n'est pas étrangère à sa faible charge polémique, est qu'elle réfute la désignation *littérature belge " tout en continuant à se référer à "notre " littérature. Cette attitude contradictoire s'inscrit dans l'ambivalence de la stratégie de dénomination/ dévalorisation dégagée ci-dessus. Elle constitue le signe par excellence de l'aporie institutionnelle dans laquelle se trouve l'écrivain belge et qui se traduit ici en ce geste double par lequel l'écrivain se nomme en désavouant son nom.

Ce qui devait être un événement dans le milieu littéraire belge ne fut en réalité que l'amorce d'un débat. L'un des signataires (Paul Fierens) a beau annoncer, peu après la publication du "Manifeste", qu'il promet "de jeter aujourd'hui quelque trouble dans le landerneau des lettres belges* et conclure qu' *il sera vraisemblablement très discuté ${ }^{26}$, à la vérité, le texte passe plutôt sans discussion, approuvé par la plupart des commentateurs, à quelques nuances près. Plusieurs importantes revues de l'époque n'en parlent guère (notamment Anthologie, Le thyrse, L'avant-

26 Paul Fierens, dans Toute l'édition, 10 juin 1937, Musée de la littérature (Bruxelles), 5818/72. 
poste), d'autres (dont Le flambeau) se contentent de citer partiellement le texte sans y ajouter de commentaires. Ce sont les signataires eux-mêmes qui feront surtout écho au "Manifeste" dans la presse; les autres réactions, plutôt pondérées, cherchant à faire la part des choses et donnant raison aux Lundistes tout en ajoutant la nécessité de ne pas être à la remorque des lettres françaises (Le soir, Le rouge et le noir), ne dépasseront pas, somme toute, le cadre éphémère de l'article journalistique ${ }^{27}$. Hors du siècle, hebdomadaire anversois apparu à la fin de 1936, est, de par son programme, l'un des lieux d'expression les plus intéressés par la stratégie des Lundistes: défendant "une attitude de véritable clerc * et, par ailleurs, "tout ce qui touche la culture française ${ }^{28}$, ce modeste journal accueille avec le plus d'enthousiasme le "Manifeste . Il entreprend dans les mois qui suivent sa parution une "Enquête sur la position des Lettres belges", dont les résultats sont cependant assez décevants: l'hebdomadaire disparaissant dès le début de 1938, l'enquête tourne court. Les réponses obtenues marquent toutes une distance par rapport au texte original; mais il ressort surtout que le "Manifeste" ne suscite guère de véritable prise de position de la part des écrivains. Le champ littéraire belge se caractérise apparemment par ceci qu'aucune position n'y est en soi défendable: avouer la sienne, c'est courir le risque de s'y voir confiné pour toujours et de ne plus être à même d'accéder à quelque consécration extra-muros beaucoup plus importante. Se situer dans l'espace belge, c'est ne pas se situer dans l'espace parisien.

27 Raphaël de Smedt a recensé la plupart des articles portant sur *le Manifeste du Lundi ", loc. cit., p. 195.

28 Hors du siècle, vol. I, $\mathrm{n}^{\circ} 1$, novembre 1936, p. 1. 